\title{
Enjeux et perspectives de l'enseignement des SIG aux géographes et aux urbanistes
}

\author{
Boris Mericskay \\ UMR Espaces et Sociétés (ESO-Rennes), Université Rennes 2 \\ Place du recteur Le Moal, 35000 Rennes, France \\ boris.mericskay@univ-rennes2.fr
}

\begin{abstract}
RÉSUMÉ. Avec les évolutions techniques et d'usages des données spatiales, l'enseignement de la géomatique se voit aujourd'hui largement questionné. Cet article revient sur les modalités pédagogiques de l'apprentissage des SIG dans les cursus de géographie et d'aménagement. Il présente une série d'enjeux relatifs aux formations spécialisées et non spécialisées.

ABSTRACT. With technical evolutions and uses of spatial data, the teaching of GIS is today largely questioned. This article reviews the pedagogical methods of learning GIS in geography and planning programs. It presents a series of issues relating to both specialized and non-specialized GIS training.

MoTS-CLÉS : SIG, enseignement, université, géographie, aménagement, pédagogie.

KEYWORDS: GIS, teaching, university, geography, planning, pedagogy.
\end{abstract}

DOI:10.3166/ISI.22.5.53-58 (C) 2017 Lavoisier

\section{Introduction}

Le développement de la géomatique comme champ opérationnel et thématique de recherche a profondément transformé les modalités d'enseignement de la cartographie comme de la géographie. En réponse à la demande grandissante du monde opérationnel en matière de compétences en SIG, les formations en géographie et en aménagement ont depuis quelques années largement introduit la géomatique dans leurs programmes sous des formes diversifiées (Ballereau et al., 2013 ; Vanara et al., 2014). Cette généralisation des cours de SIG destinés à la fois aux spécialistes et aux non spécialistes pose toute une série de questions concernant la formation des futurs professionnels des territoires. Avec la conversion de la cartographie au numérique et plus récemment au Web, les cours dédiés à la cartographie tendent à s'estomper au profit de cours méthodologiques toujours plus techniques autour de la prise en main de logiciels SIG. Dans la pratique, il est davantage question aujourd'hui de former les étudiants à des logiciels pour la 
manipulation et l'analyse de données spatiales et non plus uniquement à leur représentation sous forme de cartes statiques.

\section{Enseigner les SIG aux géographes et aux urbanistes}

La formation aux outils SIG fait aujourd'hui partie intégrante des formations initiales en géographie comme en aménagement. Ces cours d'introduction ont comme objectifs de fournir aux étudiants un socle de compétences techniques et méthodologiques pour la gestion, l'analyse et la représentation de données spatiales. Mais il existe de multiples manières d'enseigner la géomatique et la cartographie aux étudiants de licence. Le parti pris peut être d'axer les contenus pédagogiques autour de la cartographie thématique (sémiologie graphique, discrétisation) ou d'explorer les méthodes et fonctionnalités de gestion et d'analyse spatiale des données géographiques (jointures, tampons, découpages, proximité, etc.).

Il parait important de ne pas mettre en opposition la cartographie et la géomatique tant les apports réciproques sur les plans conceptuels, méthodologiques et techniques sont à envisager de manière globale et progressive dans la formation des étudiants aux données spatiales. À l'image de la diversité des usages des cartes dans la société, les solutions abordées doivent au mieux refléter cette hétérogénéité et insister sur la complémentarité des environnements SIG (gestion, analyse), CAO (cartographie) et DAO (mise en forme). Par ricochet, la question du choix des solutions abordées en cours n'est pas seulement technique, car les logiciels véhiculent des choix idéologiques et pédagogiques (Chrisman, 2005). Le choix n’est jamais simple, il faut à la fois coupler fonctionnalités, intuitivité, coûts des licences et demandes du monde opérationnel afin d'éviter à la fois un confinement fonctionnel associé aux outils et un choix de solutions peu ou plus utilisées.

Avec la technicisation des enseignements, l'apprentissage des principes sousjacents aux fonctionnalités des outils constitue un enjeu pédagogique majeur. Les opérations d'analyse spatiale ou de transformation des données ne sont pas toujours acquises par les étudiants, lesquels se laissent bien souvent guider par les logiciels sans vraiment s'interroger sur ce qu'ils font. Le côté " clic bouton » des solutions comme QGIS ou ArcGIS avec leurs boites à outils fournies et intuitives, où il suffit de cliquer pour lancer un traitement complexe, est à double tranchant. D'un côté la facilité de configuration et d'exécution de traitements complexes est un vrai atout pour l'enseignement des SIG pour explorer et montrer les possibilités de ces outils, mais d'un autre, paradoxalement, cette simplicité d'utilisation amène les étudiants à trop souvent privilégier l'outil à la méthode.

L'exemple de la discrétisation, étape fondamentale dans la construction d'une carte thématique, est assez caractéristique des mutations qui s'opèrent. Pour la majorité des étudiants, même après des heures consacrées à cette question, le découpage en classes est bien trop souvent envisagé comme une opération automatique prise en charge par le logiciel. Avec les possibilités de reprojection à la 
volée des logiciels SIG, la question des systèmes de projection représente bien souvent une autre boite noire pour les étudiants. En termes d'objectifs pédagogiques, l'équilibre entre montée en compétences techniques et compréhension des méthodes n'est pas évidente à trouver dans la mesure où les publics sont toujours plus curieux et demandeurs d'explorer rapidement des fonctionnalités complexes.

\section{Former les géomaticiens de demain}

En parallèle aux cours fondamentaux dispensés aux étudiants des filières de géographie et d'aménagement, l'offre de formation à l'université concernant les SIG se matérialise aussi par des cursus dédiés (licences professionnelles et masters) et la consolidation d'une mention géomatique dans l'offre des masters. Avec les problématiques de données massives, d'ouverture et de standardisation des données, les futurs géomaticiens doivent être capables de dialoguer et faire le pont entre thématiciens, informaticiens ou statisticiens.

L'un des principaux enjeux dans ces formations se situe actuellement au niveau de la montée en compétences techniques des étudiants autour de problématiques toujours plus nombreuses à l'image des systèmes de gestion de base de données, de l'analyse statistique, des logiciels d'extraction et de transformation (ETL) et de la programmation informatique (Ballereau et al., 2013). Comprendre le fonctionnement des systèmes informatiques est un préalable, mieux encore, savoir les programmer pour les personnaliser (en Python par exemple) permet aux étudiants de développer leurs propres scripts pour par exemple automatiser une chaine de traitement ou mettre en place des fonctionnalités selon les besoins.

Avec le développement de la géomatique sur Internet (cartographie en ligne, services Web géographiques, API, infrastructures de données spatiales, etc.), les demandes du marché de l'emploi pour la mise en place de solutions internes ou de diffusion de données sont grandissantes et assez hétérogènes (géoportails, serveurs géographiques, interfaces de visualisation, etc.). La connaissance des langages informatiques (HTML, SQL, PHP, JavaScript), des solutions de publications de données (propriétaires et libres) comme des standards de diffusion des données sont aujourd'hui des incontournables dans les programmes de masters spécialisés en SIG. En outre, face aux développements et surtout aux évolutions rapides de ces technologies, les contenus pédagogiques doivent continuellement être mis à jour afin d'être toujours le plus possible en adéquation aux attentes des organisations.

Cependant, même si la dimension technique prend davantage de place, il ne faut pas perdre de vue que l'objectif de ces cursus n'est pas de former des informaticiens, mais des spécialistes de la chaîne de traitement de l'information spatiale capables à la fois de gérer des bases de données géographiques, de diffuser des données sur le Web, de mobiliser des approches d'analyse spatiale ou encore de produire un atlas cartographique. Au-delà de compétences autour de logiciels ou de langages 
informatiques, il est important que les étudiants développent une approche critique et réflexive sur les outils et les données (gestion de projet, usages, éthique, etc.).

\section{Les grands enjeux de demain pour l'enseignement des SIG à l'université}

Avec le développement des logiciels libres et plus généralement de l’open source c'est tout l'écosystème de la géomatique qui est questionné et de fait les modalités d'enseignement associées (Azzaria et al., 2013 ; Belgui et al., 2016). Pouvoir installer gratuitement des logiciels SIG sur un nombre illimité de postes et permettre aux étudiants de les télécharger sur leurs ordinateurs personnels vient transformer les modalités d'enseignement. Cet affranchissement des contraintes classiques (disponibilités salles, horaires, etc.) permet dans la pratique une plus grande marge de manœuvre pédagogique (séances en autonomie, travail à la maison). Cependant le fonctionnement même des logiciels SIG libres à l'image de QGIS, basés sur une communauté de bénévoles, questionne sur la pérennité de ces solutions logicielles d'un type particulier qui peuvent très rapidement tomber en désuétude. Il est important pour les enseignants d'être vigilants quant aux développements des projets libres mais aussi de s'y impliquer afin de les faire évoluer (signalement de bugs, traduction, développement, soutien financier, etc.).

Dans la mouvance de l'open source, la question des données géographiques ouvertes constitue un autre enjeu important en matière d'enseignement des SIG (Demirci et Schee, 2015 ; Etherington, 2016). D’un point de vue pédagogique, l'accessibilité accrue à une multitude de données référentielles et thématiques permet la mise en place de cours mobilisant des ressources en lien avec les projets professionnels des étudiants. Ces nouveaux gisements de données conduisent également à proposer des enseignements dédiés à ces questions (sources, structuration, interopérabilité, métadonnées, visualisation).

En termes de données spatiales ouvertes, OpenStreetMap (OSM) est un vecteur très dynamique dans la démocratisation des SIG. La formation à OSM dans les cursus commence à se généraliser tant le projet prend de l'ampleur dans les pratiques des professionnels (Weessies et Dotson, 2013). Il est ainsi question de former les étudiants à la fois à récupérer et à comprendre les données issues d'OSM afin qu'ils puissent prendre du recul quant à la qualité et l'utilisabilité de ses données (exhaustivité, couverture, précision, mise à jour, structuration, etc.). Audelà de l'usage de ces données il est également important d'initier les étudiants à contribuer au projet (production et mise à jour).

Du point de vue des dispositifs pédagogiques, l'enseignement à distance représente une voie d'avenir pour venir renouveler, compléter, voire remplacer les cours en présentiel de SIG. L'offre de formation à distance concernant les solutions techniques est aujourd'hui assez étoffée et diversifiée (formations payantes, formations diplômantes, MOOC, SPOC) et les universités commencent à se positionner sur le sujet en proposant des contenus et des approches pédagogiques 
innovantes. Si, sur le papier, ce type de projet semble intéressant, et particulièrement adapté à l'enseignement des SIG, la mise en place d'une formation en ligne nécessite une implication importante de la part des enseignants tant pour la conception et la production des contenus que l'animation des cours.

Au-delà de dispositifs formalisés, Internet offre surtout de nouvelles modalités d'(auto)apprentissage pour les étudiants. D’un côté la disponibilité d'une multitude de tutoriels conçus par des éditeurs de logiciels ou des enseignants permet une montée en compétences des étudiants en autonomie. De l'autre, les forums spécialisés dans les SIG illustrent bien les nouvelles dynamiques qui prennent forme entre utilisateurs de logiciels pour trouver des solutions techniques en faisant appel au collectif.

Enfin, au-delà de la familiarisation avec des outils, en se fondant sur une approche par compétences, il s’agit aussi aujourd'hui de transmettre aux étudiants les éléments d'une culture du géonumérique tant les données spatiales et les outils associés sont présents dans leur quotidien et dans leurs futures pratiques professionnelles (Bearman et al., 2016 ; Shin et al., 2016). Plusieurs thématiques à l'intersection de la géomatique et de l'aménagement du territoire sont à envisager, notamment autour des thématiques de la modélisation 3D du territoire (maquettes numériques urbaines et de bâtiments), des données territoriales (données ouvertes et données massives) et des nouvelles pratiques d’aménagement (villes intelligentes, urbanisme tactique). De vastes perspectives s'ouvrent donc en termes d'enseignement des SIG et plus largement du géonumérique à destination des spécialistes que des non spécialistes, tant la géomatique est plus globalement la donnée spatiale occupe une place grandissante dans la société.

\section{Bibliographie}

Azzaria M., Zamperlina P., Landia F. (2013). GIS in geography teaching. Journal of Research and Didactics in Geography, vol. 2-2013, p. 27-42.

Ballereau S., Bazile P., Dejour N., Dissard O., Gibson D., Isenmann M., Parisi P. (2013). Devenir, former, recruter un géomaticien : Petit guide pratique de la géomatique à destination des employeurs, des candidats et des formateurs. AFIGEO.

Belgiu M., Strobl J., Wallentin G. (2015). Open Geospatial Education. ISPRS International Journal of Geo-Information, vol. 4, n² 2, p. 697-710.

Bearman N., Jones N., Andre I. (2016) The future role of GIS education in creating critical spatial thinkers. Journal of GeoGraphy in hiGher education, vol. 40, n 3, p. 394-408.

Chrisman N. (2005). Full circle: more than just social implications of GIS. Cartographica, vol. $40, n^{\circ} 4$, p. 23-35.

Demirci A., Schee J. (2015). Geospatial Technologies and Geography Education in a Changing World. Springer, Tokyo. 
58 ISI. Volume $22-n^{\circ} 5 / 2017$

Etherington T.R. (2016). Using open-ended geographic information system assessments to allow students to construct relevant geographies given the internationalisation of tertiary education. New Zealand Geographer. Vol. 72, n² 2, p. 151-158.

Shin E., Milson A. J., Smith, T. (2016). Future teachers' spatial thinking skills and attitudes. Journal of Geography, vol. 115, n 4, p. 139-146.

Vanara N., Huet C., Payet P., Pech P., Goeldner-Gianella L. (2014). Environnement et géomatique : des métiers en mutation, EchoGéo, n ${ }^{\circ}$ 27. https://echogeo.revues.org/13790

Weessies K. W., Dotson D. S. (2013). Mapping for the Masses: GIS Lite \& Online Mapping Tools in Academic Libraries. Information Technology and Libraries, vol. 32, $\mathrm{n}^{\circ} 1$, p. 23 35. 